\title{
The intermediate coherent scattering function of entangled polymer melts : a Monte Carlo test of des Cloizeaux' theory
}

\author{
J. Wittmer (*), W. Paul and K. Binder \\ Institut für Physik, Johannes Gutenberg-Universität Mainz, Staudinger Weg 7, D-55099 Mainz, \\ Germany
}

(Received 30 July 1993, accepted in final 31 January 1994)

\begin{abstract}
Using the bond fluctuation model for flexible polymer chains in a dense melt the intermediate coherent scattering function for chains containing $N=200$ monomers is calculated and interpreted in terms of a recent theory of des Cloizeaux. The theory yields an explicit description for the crossover from the Rouse model to the regime where reptation prevails, for the limit $N \rightarrow \infty$. While the Monte Carlo data are qualitatively compatible with this description, an accurate estimation of the tube diameter is prevented due to the onset of a diffusive decay of the scattering function, not included in the theory. For a full quantitative analysis of the Monte Carlo data (as well as of experiments on chains with not extremely large molecular weight) an extension of the theory for finite $N$ would be required.
\end{abstract}

\section{Introduction and overview.}

The detailed understanding of the dynamics of flexible entangled polymers in dense melts is still a challenge [1-19]. Most popular is the concept [4-6] that chains are constrained to move anisotropically along their own contour in a tube, but this idea is not yet generally accepted [79, 11-13]. While viscoelastic measurements [3] give only a rather indirect information on the actual chain motions, these motions can be probed on microscopic scales both in space and in time by the neutron spin echo technique, which yields directly the (normalized) intermediate scattering function $S(q, t) / S(q, 0)$ [20-24], q being the wave vector of the scattering experiment and $t$ the time. From a detailed study of this function-over a wide enough range of wavelengths and times one should be able to distinguish whether the characteristic length scale $d_{\mathrm{T}}$ and associated time scale $\tau_{\mathrm{e}}$, where motions are slow in comparison with the Rouse model $[1,2,25,26]$, is caused by the onset of anisotropic motion (reptation) or other mechanisms (such as the onset of mode-coupled dynamics [11], for instance).

(*) Present address: Institute Charles Sadron, 67083 Strasbourg, France. 
Computer simulations $[12,17-20,27]$ have the distinctive advantage that they can address this problem for simplified precisely defined models where one avoids unessential complications (such as contributions to the relaxation from side group motions, polydispersity of the melt, change of the temperature distance from the glass transition when the chain length is varied, etc.) and that one obtains detailed microscopic information on all quantities of interest simultaneously. E.g., the characteristic time-scale $\tau_{\mathrm{e}}$ can be estimated also from the timedependent mean square displacement of inner monomers in the chain (Fig. 1). In addition, since the Rouse model implies $[1,2,25] g_{1}(t)=\sigma^{2}\left(W_{\text {eff }} t\right)^{1 / 2}$ where $\sigma$ is the length of the effective bond and $W_{\text {eff }}$ an « effective» Rouse rate («effective » means we have absorbed a constant prefactor of order unity in the above relation in $W_{\text {eff }}$ ), the Rouse scale $\sigma^{2} W_{\text {eff }}^{1 / 2}$ can be independently estimated, unlike experiments [20-24] where all such parameters must be extracted from $S(q, t) / S(q, 0)$ itself.

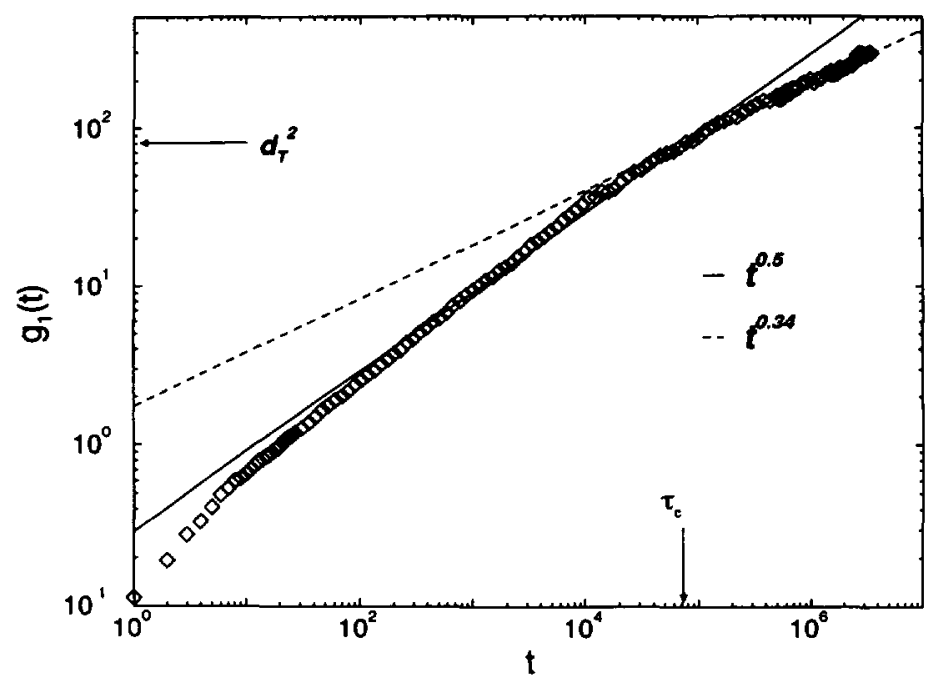

Fig. 1. - Mean-square displacement of the inner monomers, $g_{1}(t) \equiv\left\langle\left[\mathbf{r}_{1}(t)-\mathbf{r}_{t}(0)\right]^{\hat{}}\right\rangle$, displayed on a $\log -\log$ plot versus time for a polymer melt modelled by the bond fluctuation model on the simple cubic lattice for a volume fraction $\phi=0.5$ of occupied sites, chainlength $N=200$, and temperature $T=1$ (temperature enters the model through a potential energy $E(\alpha)=\varepsilon \cos \alpha$ for the angle $\alpha$ between successive bonds along a chain with $\left.\varepsilon / k_{\mathrm{B}} \equiv 1\right)$. Time is measured in units of attempted Monte Carlo Steps (MCS) per effective monomer. One can clearly distinguish the crossover from Rouse-like motion, $g_{1}(t) \propto t^{0}$, indicated by a full straight line, to a slower motion (broken straight line indicates a behavior $\left.g_{1}(t) \propto t^{034}\right)$. Arrows indicate estimation of $\tau_{\mathrm{e}}$ and $d_{\mathrm{T}}^{2}$. From Wittmer et al. [19].

Until recently the analysis of $S(q, t) / S(q, 0)$ from both experiment [20-24] and simulation $[17,19,20,28]$ was hampered by the fact that reptation theory only considered the limiting cases [6] $q d_{\mathrm{T}} \ll 1, t \gg \tau_{\mathrm{e}}$. i.e. the theory did not include the Rouse limit [26] which applies for the opposite cases $\left(t \ll \tau_{\mathrm{e}}\right.$ or $q d_{\mathrm{T}} \gg 1$, respectively). Since both experimental data [20-24] and corresponding simulations [17-19] probe the regime around the crossover $\left(q^{-1}\right.$ and $d_{\mathrm{T}}$ are of the same order, $t$ and $\tau_{\mathrm{e}}$ are also of the same order) the limit considered by de Gennes [6] was of limited use for the interpretation of these data, and hence rather comparisons have been made mostly with the approximate theory of Ronca [7]. The validity of this 
semiphenomenological approach, however, is somewhat uncertain, and hence case of poor agreement reported in the literature [24] need not imply a deficiency in the reptation concept.

Distinctive progress was recently made by des Cloizeaux [16] who calculated $S(q, t) / S(q, 0)$ from reptation theory in the limit $N \rightarrow \infty$ where the entanglement constraints can be treated as fixed. His theory shows distinct deviations from Ronca's treatment [7], but comparison with the experimental data [24] on polybutadiene is rather encouraging. In the present paper, we hence wish to check this theory [16] further by reanalyzing the "data " on $S(q, t) / S(q, 0)$ obtained from Monte Carlo simulations of the bond fluctuation model [19]. In this study, a reasonable fit to the Ronca [7] prediction has been obtained (Fig. 2). If this good fit implied a real discrepancy with des Cloizeaux' predictions [16], this would clearly raise doubts on the validity of reptation concepts for the simulated model. In section 2 we hence recall the main theoretical results, while in section 3 we present a comparison of numerical evaluations of his formula [16] (unfortunately these are very tedious and hence their use in a fitting procedure is not straightforward) with the simulation results. This comparison reveals that this theory is also qualitatively compatible with the simulations, but not useful for their quantitative analysis due to the restrictive assumption of the limit $N \rightarrow \infty$ (i.e., $N / N_{\mathrm{e}} \rightarrow \infty$ where $N_{\mathrm{e}}$ is the number of monomers between "entanglements »). This means that the diffusive decay time of the mesh of entanglements, $\tau_{\text {mesh }}$, has to be very large compared to the entanglement time, $\tau_{\text {mesh }} \gg \tau_{\mathrm{e}}$.

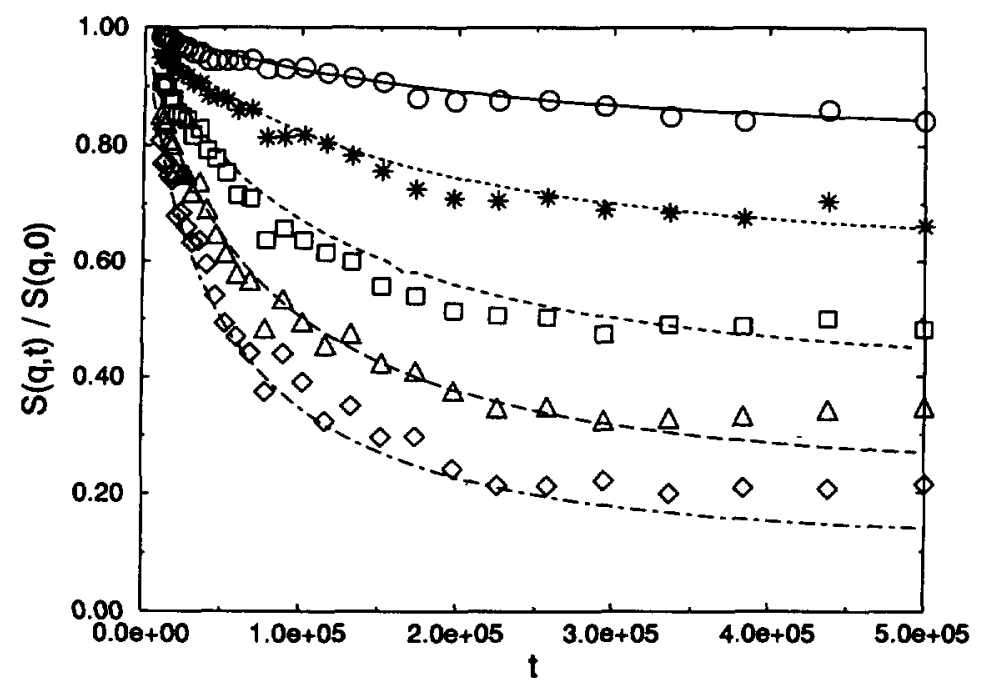

Fig. 2. - Fit of the Ronca prediction (curves) for the dynamic scattering factor $S(q, t) / S(q, 0)$ to the Monte Carlo simulation data (symbols) of the same model as specified in figure 1 , for several choices of $q$ (being measured in units of the inverse lattice constant) $: q=0.15$ (circles), 0.2 (stars), 0.25 (squares), 0.3 (triangles) and 0.35 (diamonds), respectively. From Wittmer et al. [19].

\section{Theoretical background.}

The coherent scattering function of one polymer containing $N$ monomers in a melt is defined as $[2,6]$

$$
S(\mathbf{q}, t)=\frac{1}{N} \sum_{i=1}^{N} \sum_{j=1}^{N}\left\langle\exp \left\{i \mathbf{q} \cdot\left[\mathbf{r}_{l}(t)-\mathbf{r}_{j}(0)\right]\right\}\right\rangle
$$


Here $\langle\cdots\rangle$ means a statistical average over all configurations of the chain in thermal equilibrium. des Cloizeaux [16] assumes that in the limit $N \rightarrow \infty$ and $t$ of order $\tau_{\mathrm{e}}$ that he wishes to consider, the displacements $\mathbf{r}_{,}(t)-\mathbf{r}_{j}(0)$ can be considered as random Gaussian variables, such that $\left\langle\exp \left\{i \mathbf{q} \cdot\left[\mathbf{r}_{,}(t)-\mathbf{r}_{j}(0)\right]\right\}\right\rangle=\exp \left\{-\frac{q^{2}}{6}\left\langle\left[\mathbf{r}_{1}(t)-\mathbf{r}_{j}(0)\right]^{2}\right\rangle\right\}$, and that the polymer can be divided into branches in between « entanglement points » which are rigidly fixed and thus these branches are completely independent from each other. With some algebra, des Cloizeaux [16] obtains from these assumptions the intermediate scattering function as $\left[Z \equiv q^{2} d_{\mathrm{T}}^{2} / 6, X \equiv q^{2} \sigma^{2}\left(\frac{\pi}{36} W t\right)^{1 / 2}, W\right.$ being the Rouse rate $]$

$$
S(q, t) / S(q, 0)=\frac{1}{Z} \ln (1+Z)+\int_{0}^{\infty} \mathrm{d} y \mathrm{e}^{-y} F(X, y Z)
$$

where

$$
\begin{aligned}
F(X, z)=z \int_{0}^{1} \mathrm{~d} A \int_{0}^{1} \mathrm{~d} B \exp [ & -z A-\frac{1}{\sqrt{\pi}} \int_{0}^{x} \mathrm{~d} u \sum_{p=-\infty}^{p=+\infty} \times \\
& \left.\times\left\{\exp \left[-\frac{(A-2 p)^{2} z^{2}}{u^{2}}\right]-\exp \left[-\frac{(B-2 p)^{2} z^{2}}{u^{2}}\right]\right\}\right] .
\end{aligned}
$$

The integral in equation (2) means that a random Poisson distribution of the distances between entanglement points has been assumed, i.e. $d_{\mathrm{T}}^{2}$ here has already the meaning of a correspondingly averaged quantity. Note that in the limit $Z \rightarrow \infty$ the simple Rouse model results [26]

$$
S(q, t) / S(q, 0)=\int_{0}^{\infty} \mathrm{d} a \exp \left[-a-\frac{X}{\sqrt{\pi}} \int_{0}^{1} \mathrm{~d} u \exp \left(-\frac{a^{2}}{X^{2} u^{2}}\right)\right],
$$

while Ronca's result [7] is

$$
S(q, t) / S(q, 0)=\frac{1}{4} Z \int_{0}^{\infty} \exp \left\{-\frac{1}{8} Z g\left(\omega, 36 X^{2} / Z^{2}\right)\right\} \mathrm{d} \omega
$$

where the function $g$ is defined in terms of the complementary error function erfc as

$$
g(w, u)=2 w-\exp (w) \operatorname{erfc}\left(\frac{w}{2 \sqrt{u}}+\sqrt{u}\right)+\exp (-w) \operatorname{erfc}\left(\frac{w}{2 \sqrt{u}}-\sqrt{u}\right) .
$$

Both equations (2), (5) cannot be simplified further but need be evaluated numerically. A simplification occurs only in the limit $X \rightarrow \infty$ where both equations (2), (5) yield a saturation at a nonzero plateau value (while the actual structure factor for finite chain length decays to zero in this limit, of course). In the framework of the reptation model, this decay reflects the renewal of the tube. These plateaus are described by

$$
\begin{aligned}
& S(q, \infty) / S(q, 0)=\frac{1}{Z} \ln (1+Z)-\frac{1}{2} Z \int_{0}^{1} \mathrm{~d} x \frac{\ln (2 x-1)}{(1+Z x)^{2}}, \quad \text { des Cloizeaux }, \\
& S(q, \infty) / S(q, 0)=\frac{1}{4} Z \int_{0}^{\infty} \mathrm{d} v \exp \left[-\frac{Z}{4}(v+\exp (v))\right], \quad \text { Ronca . }
\end{aligned}
$$

\section{Comparison of des Cloizeaux' theory with the simulations and discussion.}

When we compare the data of figure 2 (where the curves shown represent equation (5) with $d_{\mathrm{T}}=24.5$ and $W=1.2 \times 10^{-4}$ as fitted parameters, the rate $W$ being taken from the mean- 
square displacement $g_{1}(t)$, Fig. 1) to theoretical predictions, we must note that we cannot use $d_{\mathrm{T}}$ as extracted in figure 1 for the quantity $Z=q^{2} d_{\mathrm{T}}^{2} / 6$, since the $d_{\mathrm{T}}$ used in des Cloizeaux' theory is only of the same order of magnitude as the $d_{\mathrm{T}}$ seen in $g_{1}(t)$, but certainly differs from it quantitatively by a numerical factor. Of course, it would be desirable to compute both $g_{1}(t)$ and $S(q, t)$ from the same theory because this would then allow a meaningful comparison between the corresponding simulation results as well. However, the description of entanglement constraints as fixing rigidly monomers along the chain (which are an average distance $N_{\mathrm{e}}$ along the backbone apart from each other) necessarily implies that $g_{1}\left(t \gg \tau_{\mathrm{e}}\right) \propto d_{\mathrm{T}}^{2}$, independent of $t$, because the monomers then cannot escape arbitrarily far from the rigidly fixed entanglement points. The assumptions used in reference [16] thus are not immediately suitable to calculate the crossover of $g_{1}(t)$ (or of the incoherent scattering function).

Maintaining thus the above estimate for $W$ and the quantity $\sigma$, which is extracted from simulation results for static quantities, see reference [19], we can transform the simulation times to the rescaled variable $X=q^{2} \sigma^{2}\left(\frac{\pi}{36} W t\right)^{1 / 2}$, while $Z$ has to be treated as an adjustable parameter. Thus figure 3 compares simulation data for three representative values of $q$ with equation (2) which was evaluated for a wide regime of $Z$. While for $X \leq 1$ the data for all $q$ clearly superimpose, as it must be in this plot due to the choice of a Rouse scaling variable, the data level off from the Rouse function (which is the limiting curve for large $Z$ ), indicating compelling evidence for the onset of slower relaxation. Qualitatively, this plot is very similar to the comparison of des Cloizeaux [16] (his Fig. 9) with the experimental data of Richter et al. [24]. However, we do not attempt a quantitative fitting for several reasons : (i) the statistical errors in the data are rather large ; (ii) there are systematic errors in $Z$ to be expected, since the finite chain length $N$ leads to pronounced deviations from equation (2). At a time of order

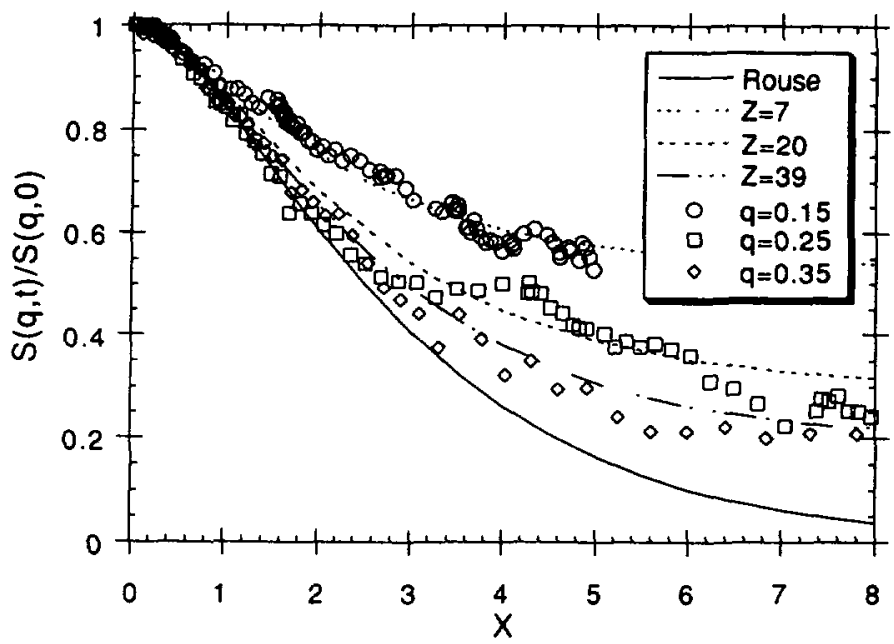

Fig. 3. - Dynamic scattering function $S(q, t) / S(q, 0)$ plotted is. the Rouse variable. $X=$ $q^{2} \sigma^{2}\left(\frac{\pi}{36} W t\right)^{1 / 2}$ Simulation data for the same model as in figures 1,2 are included for three values of $q=0.15$ (circles), 0.25 (squares), 0.35 (diamonds). Note that all three values should superimpose on a single curve, given by equation (4), if the Rouse model were valid (which is shown as full curve). Broken curves show predictions of the des Cloizeaux theory [16] for several choices of $Z: Z=7$ (dotted curve), $Z=20$ (broken curve), and $Z=39$ (dash-dotted curve). 
$\tau_{\mathrm{N}} \propto W^{-1} N^{3} / N_{\mathrm{e}}$ the plateau described by equation (7) must have decayed to zero, although $\tau_{N}$ is probably of the order of $10^{8} \mathrm{MCS}$ in our case (cf. Ref. [18]), and only data up to $t=5 \times 10^{5} \mathrm{MCS}$ are included in figure 3, some systematic deviations are to be expected. More importantly, also the parameter $q R_{\mathrm{g}}$ where $R_{\mathrm{g}}$ is the gyration radius of the chains needs to be included in the theory, and for the chosen values of $q$ it is not negligibly small. From the experience with mean square displacements such as $g_{1}(t)$ (Fig. 1) for different chain lengths one finds that in the considered time window curves for different $N$ practically superimpose for $t \leq \tau_{\mathrm{e}}$ and then the displacements for the smaller values of $N$ (but still in the entangled regime) are only slightly larger than for $N \rightarrow \infty$ [17-19]. Due to these effects, it is not unreasonable to assume that the data for finite $N$ should fall slightly below the corresponding theoretical curves for $N \rightarrow \infty$. Therefore some systematic errors due to the finiteness of $N$ must be expected, but quantitative theoretical predictions for these effects are still lacking. However, if we disregard this problem and simply try to find a tube diameter that fits our data reasonably well, we find $d_{\mathrm{T}} \approx 43.7$ (yielding $Z \approx 7, Z \approx 20$ and $Z \approx 39$ for the three values of $q$ shown in Fig. 3 ). This is somewhat larger than the value obtained previously from fitting Ronca's model, $d_{\mathrm{T}}=24.5$ [19], but still a reasonable value.

Comparing figures 2, 3 one must say that on the basis of our simulations one clearly cannot discriminate between the models of Ronca [7] and des Cloizeaux [16] - within the rather large statistical and systematic errors both are compatible with the simulation data. For both models an extension taking corrections due to finite size of the chains into consideration would be highly desirable.

\section{Acknowledgments.}

We thank Professor J. des Cloizeaux for sending his preprint (Ref. [16]) and for stimulating discussions. One of us (J. W.) thanks the Bundesministerium für Forschung und Technologie (BMFT) for support under Grant $\mathrm{N}^{\circ} 03 \mathrm{M} 4040$.

\section{References}

[1] de Gennes P. G., Scaling Concepts in Polymer Physics (Cornell University Press, Ithaca, 1979).

[2] Doi M. and Edwards S. F., The Theory of Polymer Dynamics (Clarendon Press, Oxford, 1986).

[3] Ferry J. D., Viscoelastic Properties of Polymers (Wiley, New York, 1980).

[4] de Gennes P. G., J. Chem. Phys. 55 (1971) 572.

[5] Doi M. and Edwards S. F., J. Chem. Soc., Faraday Trans. 74 (1978) 1789, 1802, 1818.

[6] de Gennes P. G., J. Chem. Phys. 72 (1980) 4756.

[7] Ronca G., J. Chem. Phys. 79 (1983) 1031.

[8] Kavassalis T. A. and Noolandi J., Macromolecules 21 (1988) 2869.

[9] Fixman M., J. Chem. Phys. 89 (1988) 3892.

[10] Hess W., Macromolecules 19 (1986) $1395 ; 20$ (1987) $2589 ; 21$ (1988) 2620.

[11] Schweizer K.-G., J. Chem. Phys. 91 (1989) 5802, $5822 ;$ J. Non-Cryst. Solids 131-133 (1991) 643 ; Phys. Scripta (1993, in press).

[12] Skolnick J. and Kolinski A., Adv. Chem. Phys. 78 (1990) 223.

[13] Lodge T. P.. Rotstein N.' A. and Prager S., Adv. Chem. Phys. 79 (1990) 1.

[14] Rostiashvili V. G., Sov. Phys. JETP 70 (1990) 563.

[15] des Cloizeaux J., Europhys. Lett. 5 (1988) 437 ; 6 (1988) 475 [E] ; Macromolecules 23 (1990) $4678 ; 25$ (1992) 835.

[16] des Cloizeaux J., J. Phys. I France 3 (1993) 1523. 
[17] Kremer K., Grest G. S. and Carmesin I., Phys. Rev. Lett. 61 (1988) 566 ;

Kremer K. and Grest G. S., J. Chem. Phys. 92 (1990) 5057 ; J. Chem. Soc. Faraday Trans. 88 (1992) 1707.

[18] Paul W., Binder K., Heermann D. W. and Kremer K., J. Phys. II France 1 (1991) 37 ; J. Chem. Phys. 95 (1991) 7726.

[19] Wittmer J., Paul W. and Binder K., Macromolecules 25 (1992) 7211.

[20] Richter D., Baumgärtner A., Binder K., Ewen B. and Hayter J. B., Phys. Rev'. Lett. 47 (1981) 109 ; 48 (1982) 1695.

[21] Higgins J. S. and Roots J. E., J. Chem. Soc., Faraday Trans. 281 (1985) 757 ;

Higgins J. S., Phystca 136B (1986) 201.

[22] Richter D., Farago B. and Wagner T., Phys. Rev. Lett. 62 (1989) 2140.

[23] Richter D., Farago B., Fetters L. J., Huang J. S., Ewen B. and Lartigue C., Phys. Rev. Lett. 64 (1990) 1389.

[24] Richter D., Butera R., Fetters L. J., Huang J. S., Farago B. and Ewen B., Macromolecules 25 (1992) 6156.

[25] Rouse P. E., J. Chem. Phys. 21 (1953) 1272.

[26] de Gennes P. G., Physics (N.Y.) 3 (1967) 97.

[27] For a general review, see Binder K., in Computational Modelling of Polymers, J. Bicerano, Ed. (M. Dekker, New York, 1992) p. 221.

[28] Baumgärtner A. and Binder K., J. Chem. Phys. 75 (1981) 2994 ;

Baumgärtner A., Annu. Rev'. Phys Chem. 35 (1984) 419. 